\section{Design of Linear-Phase Recombination Nonuniform Filter Banks}

X. M. Xie, S. C. Chan, Member, IEEE, and T. I. Yuk, Member, IEEE

\begin{abstract}
This correspondence proposes a novel method for designing a class of recombination nonuniform filter banks (RNFBs) with the linearphase (LP) property. In the structure of the proposed nonuniform filter bank (FB), certain channels of an $M$-channel uniform FB are merged by synthesis filters of transmultiplexers (TMUXs), yielding nonuniform subbands. By a detailed analysis of the frequency characteristics of the RNFBs, we derive the existence and matching conditions that are necessary for constructing the proposed RNFBs having good frequency responses. With those conditions being satisfied, the design problem becomes that of a set of LP uniform FBs. By using the Parks-McClellan algorithm, we are able to design the near-perfect-reconstruction LP RNFBs in a very simple and efficient way as demonstrated by two examples.
\end{abstract}

Index Terms-Recombination nonuniform filter banks (RNFBs), linear phase (LP), near-perfect reconstruction, transmultiplexer (TMUX).

\section{INTRODUCTION}

In applications such as image, audio, and speech analysis and coding, perfect-reconstruction (PR) filter banks (FBs) with nonuniform frequency spacing usually offer considerable flexibility in frequency partitioning. A significant amount of research effort has been devoted to the theory and design of the nonuniform FBs [1]-[5]. One popular design method is the direct one where a direct structure is adopted [2]. However, this design usually involves nonlinear optimization with a considerable number of parameters. Another useful approach is the indirect method [3], where certain channels of a uniform FB are merged using the synthesis filters of transmultiplexers (TMUXs), giving rise to a near-PR recombination nonuniform FBs (RNFBs). It was shown recently in [4] that it is possible to achieve PR in this structure. A class of RNFBs based on cosine-modulated FBs (CMFBs) were also proposed. By imposing a simple matching condition on the prototype filters, we were able to obtain the recombination nonuniform CMFBs (RN-CMFBs) with low design and implementation complexities and good frequency characteristics. The advantage of the RNFB is that the $\mathrm{PR}$ property is structurally imposed as long as the original uniform FB and the recombination TMUXs are PR. Compared with another merging method in [6] where a simple transformation is used, the general recombination TMUX employed in the RNFB can help to create a single subband with good frequency characteristics.

The majority of the existing work on the nonuniform FB design cannot maintain linear-phase (LP) property, because of the high complexity associated with phase distribution. In this correspondence, we propose a novel method for designing RNFBs with LP property. To begin with, we make two assumptions related to the RNFBs. The first one is that the $M$-channel uniform FB alone and the TMUXs alone are all LP in the recombination structure. The second one is that the number of channels of the uniform FB and that of the recombination TMUX are coprime. With this assumption, it is possible for the RNFB to ob-

Manuscript received January 20, 2005; revised July 9, 2005. This work was supported in part by Nation Natural Science Fund of China under Grant no. 60372047. The associate editor coordinating the review of this manuscript and approving it for publication was Dr. Henrique Malvar.

X. M. Xie is with the School of Electronic Engineering, Xidian University, Xi'an, China (e-mail: xmxie@ see.xidian.edu.cn).

S. C. Chan and T. I. Yuk are with the Department of Electrical and Electronic Engineering, University of Hong Kong, Hong Kong, China (e-mail: scchan@eee.hku.hk; tiyuk@eee.hku.hk).

Digital Object Identifier 10.1109/TSP.2006.874400 tain linear time-invariant (LTI) analysis and synthesis filters, instead of the original linear periodic time-varying (LPTV) filters. As a result, the frequency responses of the analysis filters can be optimized directly and the design procedure can be simplified significantly. In this correspondence, we first study the frequency characteristics of the analysis filters of the proposed LP RNFBs. Based on the analysis results, the existence and the matching conditions for obtaining LP RNFBs with good filter quality are then derived. With those conditions being satisfied, the design of the LP nonuniform FBs becomes that of a set of LP uniform FBs, and thus the design procedures of the LP uniform FBs are greatly simplified. Rather than involving the LP paraunitary FB [7] and the LP FBs by cosine and sine modulation [8], the LP uniform FBs introduced in this correspondence are obtained by using the well-known Parks-McClellan algorithm with transition bands having cosine roll-off type.

The rest of this correspondence is organized as follows. The frequency characteristics of RNFBs are analyzed in Section II. The existence and matching conditions are derived in Section III, followed by the procedures and examples in Section IV and conclusions in Section V.

\section{FREQUENCY CHARACTERISTICS OF RECOMBINATION NONUNIFORM FILTER BANKS}

Before presenting a detailed analysis of the frequency characteristics of the RNFBs, let us give a brief introduction on the RNFBs.

The structure of an $L$-band RNFB is shown in Fig. 1. In this structure, $m_{l}$ subchannels of an $M$-channel uniform FB are merged by an $m_{l}$-channel TMUX, where $l=0, \cdots, L$, thereby producing nonuniform subbands with a sampling rate of $m_{l} / M$. At the synthesis section, the analysis filters of the TMUX are used to produce the $m_{l}$ subbands. Similar merging can be performed for other consecutive channels. Notice that the modulation operation associated with the constants $c_{0} \sim c_{M-1}$ and $c_{0}^{-1} \sim c_{M-1}^{-1}$ do not affect the PR condition. They are introduced to suppress the undesirable spurious response, which we will explain later in Section III. It was observed in [4] that if the uniform FB and the TMUXs are PR, then the nonuniform FB will also be PR. This is because if the input signals of the TMUX as indicated by the dotted line in Fig. 1 can be approximately reconstructed at the output of the TMUX, the merging operation is equivalent to the introduction of a certain delay in the $m_{l}$ channels. If these delays are compensated in other branches, the whole structure is PR. This structural PR property simplifies the design of the nonuniform FB, which was already demonstrated by the RN-CMFBs in [4] and [5].

Because the analysis filters of the RNFB are in general LPTV, their design can be complicated. Fortunately, if $M$ and $m_{l}$ are coprime, it is possible to come up with an LTI representation of the analysis filters by interchanging the order of the decimator and interpolator [4]. With this coprime assumption in mind, we will examine in some details the frequency characteristics of the analysis filters in the rest of this section.

To begin with, let us study the equivalent LTI representation of the RNFB. Consider the merging of the subbands in Fig. 1. If $m_{l}$ and $M$ are coprime, then the decimator $(M)$ and the interpolator $\left(m_{l}\right)$ can be interchanged. Moreover, using the noble identity [9], $H_{r_{l}+i}(z)$ and $G_{l, i}(z), i=0, \ldots, m_{l}-1$ can be moved across the interpolator and decimator, where $r_{l}$ are the starting index of the merged channels of the $M$-channel uniform FB. It gives the following equivalent LTI representation of the analysis filter:

$$
\left.\hat{H}_{l}(z)=\sum_{i=0}^{m_{l}-1} c_{r_{l}+i} H_{r_{l}+i}^{(} z^{m_{l}}\right) G_{l, i}\left(z^{M}\right) .
$$




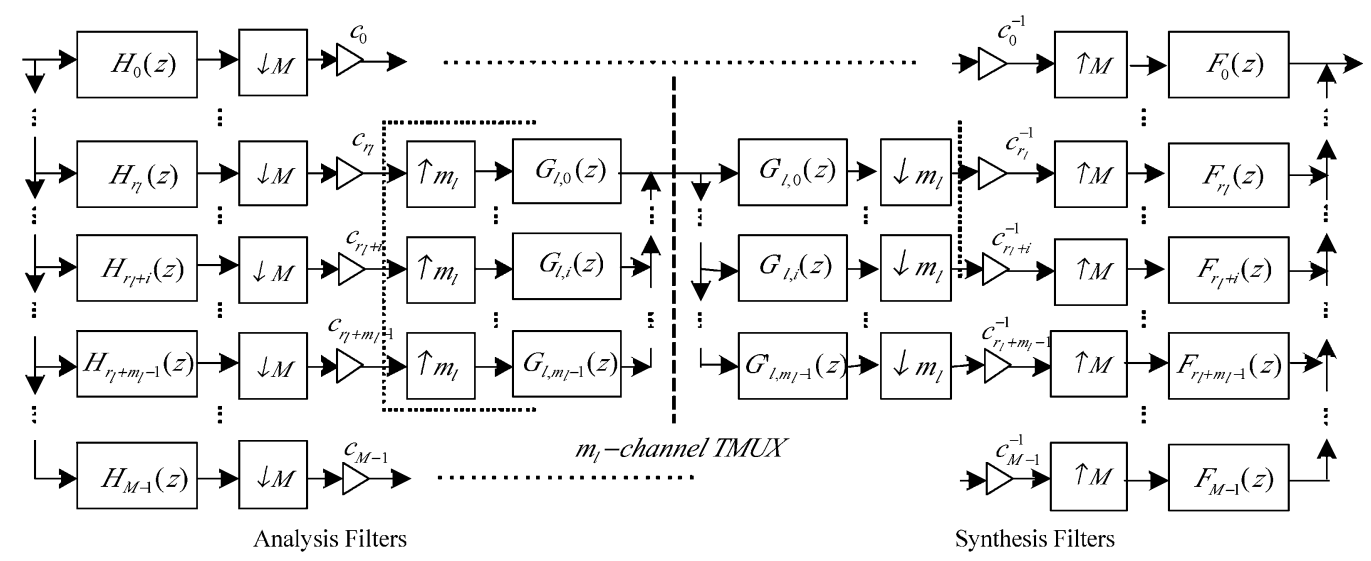

Fig. 1. Structure of linear-phase recombination nonuniform filter banks.

It should be noticed that if the starting index of the merged channel is odd, the inputs of the TMUX have to be modulated by the sequence $(-1)^{n}$ in the corresponding channels [2] so as to overcome the spectral inversion, an inherent problem of nonuniform FBs. It can be verified that the modulator of $(-1)^{n}$ commutes either with the up-sampler or with the down-sampler so that the analysis filters can be represented as the cascade of certain LTI filter with the modulator.

Consider the interpolated filter $G_{l, i}\left(z^{M}\right)$ in (1). Here, we assume that the $m_{l}$-channels to be merged are taken from the $M$-channel uniform FB with the channel index $r_{l}, r_{l}+1, \cdots, r_{l}+m_{l}-1$. The merging operation is performed by employing the synthesis filters $G_{l, i}(z)$ of the $m_{l}$-channel TMUX. Since the PR condition of a uniform TMUX is identical to that of a 1-skewed FB, the TMUX can be derived from a 1 -skewed FB [10]. Let $\left\{G_{l, i_{\perp} \mathrm{FB}}^{\prime}(z), G_{l, i \_\mathrm{FB}}(z)\right\}$ and $\left\{G_{l, i}^{\prime}(z), G_{l, i}(z)\right\}, i=0, \cdots, m_{l}-1$ be the analysis/synthesis filter pairs of a uniform FB and a 1-skewed FB respectively. Then, we have $G_{l, i}^{\prime}(z)=G_{l, i \_F B}^{\prime}(z)$ and $G_{l, i}(z)=z^{-1} G_{l, i_{-} \mathrm{FB}}(z)$. In other words, if $G_{l, i}(z)$ and $G_{l, i}^{\prime}(z)$ in Fig. 1 are the synthesis and analysis filters of a 1-skewed $m_{l}$-channel FB, then they constitute a TMUX. For notational convenience, we shall use $H(\omega)$ to denote $H\left(e^{j \omega}\right)$ in the rest of the paper. By using $G_{l, i}(\omega)=e^{-j \omega} G_{l, i}$ FB $(\omega)$, (1) becomes

$$
\begin{aligned}
\hat{H}_{l}(\omega) & =\sum_{i=0}^{m_{l}-1} c_{r_{l}+i} H_{r_{l}+i}\left(m_{l} \omega\right) G_{l, i}(M \omega) \\
& =e^{-j \omega} \sum_{i=0}^{m_{l}-1} c_{r_{l}+i} H_{r_{l}+i}\left(m_{l} \omega\right) G_{l, i \neq \mathrm{FB}}(M \omega) \\
& =e^{-j \omega} D(\omega)
\end{aligned}
$$

where

$$
D(\omega)=\sum_{i=0}^{m_{l}-1} c_{r_{l}+i} H_{r_{l}+i}\left(m_{l} \omega\right) G_{l, i+\mathrm{FB}}(M \omega) .
$$

Let

$$
\begin{aligned}
H_{r_{l}+i}\left(m_{l} \omega\right) & =A_{r_{l}+i}(\omega)=A_{r_{l}+i}^{+}(\omega)+A_{r_{l}+i}^{-}(\omega) \\
G_{l, i \_F B}(M \omega) & =B_{i}(\omega)=B_{i}^{+}(\omega)+B_{i}^{-}(\omega)
\end{aligned}
$$

where $A_{r_{l}+i}^{+}(\omega)$ and $A_{r_{l}+i}^{-}(\omega)$ correspond to the responses of $A_{r_{l}+i}(\omega)$ for positive and negative values of $\omega$ in $\left[-\pi / m_{l}, \pi m_{l}\right]$, respectively. Since the periods of $H_{r_{l}+i}(\omega)$ and $G_{l, i \text { FB }}(\omega)$ are $2 \pi$, the period of $A_{r_{l}+i}(\omega)$ is $2 \pi m_{l}$ and that of $B_{i}(\omega)$ is $2 \pi M$. Similarly, $B_{i}^{+}(\omega)$ and $B_{i}^{-}(\omega)$ correspond to the responses of $B_{i}(\omega)$ in $[-\pi M, \pi / M]$.

Our objective is to study the conditions on $H_{r_{l}+i}(\omega)$ and $G_{l, i} \mathrm{FB}(\omega)$ such that $\hat{H}_{l}(\omega)$ will possess the desirable frequency characteristics. To simplify the analysis, we assume that 1) the stopband cutoff frequencies of $H_{r_{l}+i}(\omega)$ lie within $\left[\left(\left(r_{l}+i\right) \pi / M-\pi /(2 M)\right),\left(\left(r_{l}+i+1\right) \pi / M+\pi(2 M)\right)\right]$ and 2) the stopband attenuation is sufficiently high that it can be treated as zero outside the region. Hence, only adjacent channels overlap in their magnitude responses.

Those assumptions also apply to $G_{l, i \mp \mathrm{FB}}(\omega)$ with the stopband cutoff frequencies in the range $\left[\left(i \pi / m_{l}-\pi /\left(2 m_{l}\right)\right),\left((i+1) \pi / m_{l}+\right.\right.$ $\left.\left.\pi /\left(2 m_{l}\right)\right)\right]$.

We now turn to $D(\omega)$ in (3). Substituting (4a) and (4b) into (3), we get $D(\omega)=\sum_{j=1}^{4} D_{j}(\omega)$, where

$$
\begin{aligned}
& D_{1}(\omega)=\sum_{i=0}^{m_{l}-1} c_{r_{l}+i} A_{r_{l}+i}^{+}(\omega) B_{i}^{+}(\omega) \\
& D_{2}(\omega)=\sum_{i=0}^{m_{l}-1} c_{r_{l}+i} A_{r_{l}+i}^{+}(\omega) B_{i}^{-}(\omega) \\
& D_{3}(\omega)=\sum_{i=0}^{m_{l}-1} c_{r_{l}+i} A_{r_{l}+i}^{-}(\omega) B_{i}^{+}(\omega) \\
& D_{4}(\omega)=\sum_{i=0}^{m_{l}-1} c_{r_{l}+i} A_{r_{l}+i}^{-}(\omega) B_{i}^{-}(\omega) .
\end{aligned}
$$

It is noticed that only the terms $D_{1}(\omega)$ and $D_{2}(\omega)$ need to be studied as given below since $D_{3}(\omega)$ and $D_{4}(\omega)$ are their conjugates.

To well analyze the terms $D_{1}(\omega)$ and $D_{2}(\omega)$, we focus on their frequency support. Denote the frequency support of a function $Q(\omega)$ as $\operatorname{Supp}(Q)$, that is, if $\omega \notin \operatorname{Supp}(Q)$, then $Q(\omega)=0$. It can be shown that

$$
\begin{aligned}
\operatorname{Supp} & \left(H_{r_{l}+i}(\omega)\right) \\
= & {\left[2 p \pi+\left(\left(r_{l}+i\right) \frac{\pi}{M}-\frac{\pi}{2 M}\right), 2 p \pi\right.} \\
& \left.+\left(\left(r_{l}+i+1\right) \frac{\pi}{M}+\frac{\pi}{2 M}\right)\right] \\
\cup & {\left[2 p \pi-\left(\left(r_{l}+i+1\right) \frac{\pi}{M}+\frac{\pi}{2 M}\right), 2 p \pi\right.} \\
& \left.-\left(\left(r_{l}+i\right) \frac{\pi}{M}-\frac{\pi}{2 M}\right)\right] \\
\operatorname{Supp}( & \left.G_{l, i \mathrm{FB}}(\omega)\right) \\
= & {\left[2 q \pi+\left(i \frac{\pi}{m_{l}}-\frac{\pi}{2 m_{l}}\right), 2 q \pi\right.} \\
& \left.+\left((i+1) \frac{\pi}{m_{l}}+\frac{\pi}{2 m_{l}}\right)\right] \\
\cup & {\left[2 q \pi-\left((i+1) \frac{\pi}{m_{l}}+\frac{\pi}{2 m_{l}}\right), 2 q \pi\right.} \\
& \left.-\left(i \frac{\pi}{m_{l}}-\frac{\pi}{2 m_{l}}\right)\right]
\end{aligned}
$$


where $p$ and $q$ are integers. Since the period of $H_{r_{l}+i}\left(m_{l} \omega\right) G_{l, i_{-} \mathrm{FB}}(M \omega)$ is $2 \pi$, we need only focus on the range $-\pi \leq \omega \leq \pi$. We notice that $p$ and $q$ satisfy

$$
|p| \leq\left\lfloor m_{l} / 2\right\rfloor \text { and }|q| \leq\lfloor M / 2\rfloor
$$

in $-\pi \leq \omega \leq \pi$. Here, $\lfloor x\rfloor$ denotes the nearest integer less than or equal to $x$. From (4a) and (6a), we have

$$
\begin{aligned}
\operatorname{Supp}\left(A_{r_{l}+i}(\omega)\right) & =\operatorname{Supp}\left(A_{r_{l}+i}^{+}(\omega)\right) \cup \operatorname{Supp}\left(A_{r_{l}+i}^{-}(\omega)\right) \\
& =\left\{\bigcup_{p} x_{p, r_{l}+i}^{+}\right\} \cup\left\{\bigcup_{p} x_{p, r_{l}+i}^{-}\right\}
\end{aligned}
$$

where

$$
\begin{aligned}
x_{p, r_{l}+i}^{+}= & {\left[\frac{2 p \pi}{m_{l}}+\left(r_{l}+i-\frac{1}{2}\right) \frac{\pi}{m_{l} M}, \frac{2 p \pi}{m_{l}}\right.} \\
& \left.+\left(r_{l}+i+\frac{3}{2}\right) \frac{\pi}{m_{l} M}\right] \\
= & {\left[s_{p, r_{l}+i}^{+}, t_{p, r_{l}+i}^{+}\right], } \\
x_{p, r_{l}+i}^{-}= & {\left[\frac{2 p \pi}{m_{l}}-\left(r_{l}+i+\frac{3}{2}\right) \frac{\pi}{m_{l} M}, \frac{2 p \pi}{m_{l}}\right.} \\
& \left.-\left(r_{l}+i-\frac{1}{2}\right) \frac{\pi}{m_{l} M}\right] \\
= & {\left[s_{p, r_{l}+i}^{-}, t_{p, r_{l}+i}^{-}\right], \quad p= \pm 0, \pm 1, \pm 2, \ldots . }
\end{aligned}
$$

Furthermore, by substituting (4b) into (6b), we have

$$
\begin{aligned}
\operatorname{Supp}\left(B_{i}(\omega)\right) & =\operatorname{Supp}\left(B_{i}^{+}(\omega)\right) \cup \operatorname{Supp}\left(B_{i}^{-}(\omega)\right) \\
& =\left\{\bigcup_{q} y_{q, i}^{+}\right\} \cup\left\{\bigcup_{q} y_{q, i}^{-}\right\}
\end{aligned}
$$

where

$$
\begin{aligned}
y_{q, i}^{+} & =\left[\frac{2 q \pi}{M}+\left(i-\frac{1}{2}\right) \frac{\pi}{m_{l} M}, \frac{2 q \pi}{M}+\left(i+\frac{3}{2}\right) \frac{\pi}{m_{l} M}\right] \\
& =\left[u_{q, i}^{+}, v_{q, i}^{+}\right], \\
y_{q, i}^{-} & =\left[\frac{2 q \pi}{M}-\left(i+\frac{3}{2}\right) \frac{\pi}{m_{l} M}, \frac{2 q \pi}{M}-\left(i-\frac{1}{2}\right) \frac{\pi}{m_{l} M}\right] \\
& =\left[u_{q, i}^{-}, v_{q, i}^{-}\right], \quad q= \pm 0, \pm 1, \pm 2, \ldots
\end{aligned}
$$

It can be seen that the lengths of these intervals are all equal to $I=$ $2 \pi /\left(m_{l} M\right)$.

Now we are ready to make the conclusion on $D_{1}(\omega)$ and $D_{2}(\omega)$ in terms of the $\operatorname{Supp}(Q)$.

$D_{1}(\omega): \operatorname{In} D_{1}(\omega)=\sum_{i=0}^{m_{l}-1} c_{r_{l}+i} A_{r_{l}+i}^{+}(\omega) B_{i}^{+}(\omega)$, the support of $\left\{A_{r_{l}+i}^{+}(\omega) B_{i}^{+}(\omega)\right\}$ is $\operatorname{Supp}\left(x_{p, r_{l}+i}^{+} \cap y_{q, i}^{+}\right)$. To determine whether they overlap each other, we examine the difference between the starting locations of the two intervals. From (8a) and (9a), we get

$$
s_{p, r_{l}+i}^{+}-u_{q, i}^{+}=\left(p M-q m_{l}+r_{l} / 2\right) I .
$$

For even $r_{l}$, we define an integer $r_{l}^{\prime}$ with $r_{l}^{\prime}=r_{l} / 2$. Then, from (10) we have

$$
s_{p, r_{l}+i}^{+}-u_{q, i}^{+}=\left(p M-q m_{l}+r_{l}^{\prime}\right) I .
$$

Notice that, for odd $r_{l}$, the similar derivation can be carried out and the same conclusion will be obtained. Therefore, we will only discuss the case when $r_{l}$ is even. If

$$
p M-q m_{l}+r_{l}^{\prime}=0
$$

then the two interval overlap exactly. Since $M$ and $m_{l}$ are coprime, the solution of $p$ and $q$ in (12) is unique in the range of (7). For $p$ and $q$ satisfying (12), $\left\{A_{r_{l}+i}^{+}(\omega) B_{i}^{+}(\omega)\right\}$ has the frequency support given by

$$
\operatorname{Supp}\left(A_{r_{l}+i}^{+}(\omega) B_{i}^{+}(\omega)\right)=\left[s_{p, r_{l}+i}^{+}, u_{q, i}^{+}\right]=\left[s_{p, r_{l}+i}^{+}, t_{p, r_{l}+i}^{+}\right]
$$

The corresponding frequency support of $D_{1}(\omega)$ is

$$
\begin{aligned}
\operatorname{Supp}\left(D_{1}\right)= & \bigcup_{i=0, \cdots, m_{l}-1} \operatorname{Supp}\left\{\left[s_{p, r_{l}+i}^{+}, t_{p, r_{l}+i}^{+}\right]\right\} \\
= & {\left[\frac{\pi}{2 m_{l} M}\left(4 p M+2 r_{l}-1\right),\right.} \\
& \left.\times \frac{\pi}{2 m_{l} M}\left(4 p M+2 r_{l}+2 m_{l}+1\right)\right] .
\end{aligned}
$$

The bandwidth of $D_{1}(\omega)$ in $[-\pi, \pi]$ is $\left(1+1 / m_{l}\right) \pi / M$. Note, the support is measured after the interpolator $\uparrow m_{l}$. Therefore, the support in the original rate is $\left(1+1 / m_{l}\right) m_{l} \pi / M$. An ideal filter will have a support of $m_{l} \pi / M$. It constitutes the desired passband of $\hat{H}_{l}(\omega)$.

$D_{2}(\omega)$ : Consider $D_{2}(\omega)$ with $D_{2}(\omega)=\sum_{i=0}^{m_{l}-1} c_{r_{l}+i}$ $A_{r_{l}+i}^{+}(\omega) B_{i}^{-}(\omega)$.

Again, we examine the difference between the starting locations of $A_{r_{l}+i}^{+}(\omega)$ and $B_{i}^{-}(\omega)$. From (8a) and (9b), we have

$$
s_{v, r_{l}+i}^{+}-u_{q, i}^{-}=\left(p M-q m_{l}+r_{l}^{\prime}+i+\frac{1}{2}\right) I .
$$

Because of the term (1/2) on the right-hand side of (14), the sum inside the bracket cannot be zero. Further, if

$$
p M-q m_{l}+r_{l}^{\prime}+i=0
$$

or

$$
p M-q m_{l}+r_{l}^{\prime}+i=-1
$$

then the transition bands of $A_{r_{l}+i}^{+}(\omega)$ and $B_{i}^{-}(\omega)$ can still overlap with $s_{p, r_{l}+i}^{+}-u_{q, i}^{-}= \pm I / 2$ corresponding to (15a) and (15b). These two supports in $D_{2}(\omega)$, denoted by $F_{i}^{+}$and $F_{i}^{-}$, can be expressed as the overlapping of $A_{r_{l}+i}^{+}$with $B_{i}^{-}$either on the left-hand or the right-hand sides of $A_{r_{l}+i}^{+}$. More precisely, we have

$$
\begin{aligned}
& {\left[s_{p, r_{l}+i}^{+}, t_{p, r_{l}+i}^{+}\right] \cap\left[u_{q, i}^{-}, v_{q, i}^{-}\right]=\left[s_{p, r_{l}+i}^{+}, v_{q, i}^{-}\right]=F_{i}^{+}} \\
& {\left[s_{p, r_{l}+i}^{+}, t_{p, r_{l}+i}^{+}\right] \cap\left[u_{q, i}^{-}, v_{q, i}^{-}\right]=\left[u_{q, i}^{-}, t_{p_{l}+i}^{+}\right]=F_{i}^{-}}
\end{aligned}
$$

each having a length of $\pi /\left(m_{l} M\right)$. It can be easily verified that $F_{i+1}^{+}=$ $F_{i}^{-}$. The overlapping of $A_{r_{l}+i}^{+}$with $B_{i}^{-}$will give rise to the undesirable spurious response in $D_{2}(\omega)$ as indicated in (17).

Introducing the indicator function of $F$ as $E_{F}$, i.e.,

$$
E_{F}(\omega)= \begin{cases}1, & \text { if } \omega \in F \\ 0, & \text { if } \omega \notin F\end{cases}
$$


we have $E_{F_{i+1}^{+}}=E_{F_{i}^{-}}$. In this way, $D_{2}(\omega)$ can be expressed as follows:

$$
\begin{aligned}
& D_{2}(\omega)= \sum_{k=0}^{m_{l}-1} c_{r_{l}+i} A_{r_{l}+i}^{+}(\omega) B_{i}^{-}(\omega) \\
&= \sum_{i=0}^{m_{l}-1} c_{r_{l}+i}\left(A_{r_{l}+i}^{+}(\omega) B_{i}^{-}(\omega) E_{F_{i}^{+}}\right. \\
&\left.\quad \quad \quad+A_{r_{l}+i}^{+}(\omega) B_{i}^{-}(\omega) E_{F_{i}^{-}}\right)
\end{aligned}
$$

The term, $\quad \sum_{i=0}^{m_{l}-2}\left(c_{r_{l}+i} A_{r_{l}+i}^{+}(\omega) \quad B_{i}^{-}(\omega) E_{F_{i}^{-}}+c_{r_{l}+i+1}\right.$ $\left.A_{r_{l}+i+1}^{+}(\omega) B_{i+1}^{-}(\omega) E_{F_{i+1}^{+}}\right)$, which we call the "cross-term," is the source of the spurious response. More specifically, it causes bumpings in the stopband of $\hat{H}_{l}(\omega)$ in (1), which was first observed in [4] for RN-CMFBs. It can be verified that the frequency interval $\operatorname{Supp}\left(D_{2}(\omega)\right)-F_{0}^{+}-F_{m-1}^{-}$constitutes the stopband of $\hat{H}_{l}(\omega)$. In what follows, we will establish the condition such that the spurious response in (17) can be forced to zero. This is achieved by imposing an appropriate value on the multiplication constant $c_{r_{l}+i}$ and a matching condition of the two filters $H_{r_{l}+i}(\omega)$ and $G_{l, i, F B}(\omega)$ in the LP RNFB.

\section{CONDITIONS FOR LINEAR-PHASE RECOMBINATION NONUNIFORM FILTER BANKS}

Before proceeding to the selection of $c_{r_{l}+i}$ and the discussion of the matching condition, let us briefly review some basic properties of LP filters. If an LP filter $H(\omega)$ has a symmetric (antisymmetric) impulse response $h(n)$, then we say $H(\omega)$ is symmetric (antisymmetric). For $H_{r_{l}+i}\left(m_{l} \omega\right)$ and $G_{l, i \_}$BB $(M \omega)$ (see (4a) and (4b)), if $A_{r_{l}+i}(\omega)$ and $B_{i}(\omega)$ are symmetric, then we have

$$
\begin{gathered}
A_{r_{l}+i}^{+}(\omega)=e^{-j \alpha \omega}\left|A_{r_{l}+i}^{R}(\omega)\right| \\
B_{i}^{+}(\omega)=e^{-j \beta \omega}\left|B_{i}^{R}(\omega)\right| \\
A_{r_{l}+i}^{-}(\omega)=e^{-j \alpha \omega}\left|A_{r_{l}+i}^{R}(\omega)\right| \\
B_{i}^{-}(\omega)=e^{-j \beta \omega}\left|B_{i}^{R}(\omega)\right| .
\end{gathered}
$$

Similarly, if $A_{r_{l}+i}(\omega)$ and $B_{i}(\omega)$ are antisymmetric, we have

$$
\begin{gathered}
A_{r_{l}+i}^{+}(\omega)=j e^{-j \alpha \omega}\left|A_{r_{l}+i}^{R}(\omega)\right| \\
B_{i}^{+}(\omega)=j e^{-j \beta \omega}\left|B_{i}^{R}(\omega)\right| \\
A_{r_{l}+i}^{-}(\omega)=-j e^{-j \alpha \omega}\left|A_{r_{l}+i}^{R}(\omega)\right| \\
B_{i}^{-}(\omega)=-j e^{-j \beta \omega}\left|B_{i}^{R}(\omega)\right|
\end{gathered}
$$

where $A_{r_{l}+i}^{R}(\omega)$ and $B_{i}^{R}(\omega)$ are the amplitude responses, $\alpha=m_{l} N_{M} / 2$ and $\beta=M N_{m_{l}} / 2 . N_{M}$ and $N_{m_{l}}$ are the filter lengths of the $M$-channel FB and the $m_{l}$-channel TMUX, respectively.

Note that, in $M$-channel LP FBs, the analysis filters are always having alternate symmetry (i.e., alternative symmetric and anti-symmetric). In our correspondence, we assume that $A_{r_{l}+i}(\omega)$ and $B_{i}(\omega)$ are symmetric when $i$ is even, and antisymmetric when $i$ is odd. The same assumption is applied to $H_{r_{l}+i}(\omega)$ and $G_{l, i}$ FB $(\omega)$. Next, we will derive the condition on the constant $c_{r_{l}+i}$ with which the existence of LP RNFBs with good frequency responses is ensured. Let us start with the analysis of $D_{1}(\omega)$ in terms of the passband flatness and then proceed to the analysis of $D_{2}(\omega)$ in terms of the spurious response suppression in the stopband.

Passband Flatness: $D_{1}(\omega)$ can be further written by (20), shown at the bottom of the page. As mentioned earlier, $D_{1}(\omega)$ constitutes the passband of $\hat{H}_{l}(\omega)$. Therefore, at the transition band of $A_{r_{l}+i}^{+}(\omega)$ and $B_{i}^{+}(\omega)$, the term inside the bracket of (20) should add up to a constant. To this end, $c_{r_{l}+i}$ and $c_{r_{l}+i+1}$ should satisfy

$$
c_{r_{l}+i}=-c_{r_{l}+i+1} .
$$

Spurious Response Suppression: As mentioned earlier, the support of $D_{2}(\omega)$, except $F_{0}^{+}$and $F_{m-1}^{-}$, corresponds to the stopband of $\hat{H}_{l}(\omega)$, and the cross-term might appear. Recall (17), which is an expression of $D_{2}(\omega)$. The term inside the bracket $(\cdot)$ can be written as

$$
\begin{aligned}
& c_{r_{l}+i} A_{r_{l}+i}^{+} B_{i}^{-} E_{F_{i}^{-}}+c_{r_{l}+i+1} A_{r_{l}+i+1}^{+} B_{i+1}^{-} E_{F_{i+1}^{+}} \\
& =e^{-j(\alpha+\beta) \omega}\left(\begin{array}{c}
c_{r_{l}+i}\left|A_{r_{l}+i}^{R}(\omega)\right|\left|B_{i}^{R}(\omega)\right| \\
+c_{r_{l}+i+1}\left|A_{r_{l}+i+1}^{R}(\omega)\right|\left|B_{i+1}^{R}(\omega)\right|
\end{array}\right) .
\end{aligned}
$$

In order to eliminate the cross-term (22), we need to choose $c_{r_{l}+i}=$ $-c_{r_{l}+i+1}$, a condition that is exactly same as (21). It is evident that to meet both the requirements of "passband flatness" and "spurious response suppression," $c_{r_{l}+i}$ and $c_{r_{l}+i+1}$ must have the relation of (21). We refer to (21) as the existence condition with which good frequency characteristics of LP LTI RNFBs can be warranted.

Having satisfied the condition in (21), (22) becomes

$$
\begin{aligned}
c_{r_{l}+i} A_{r_{l}+i}^{+} B_{i}^{-} E_{F_{i}^{-}}+c_{r_{l}+i+1} A_{r_{l}+i+1}^{+} B_{i+1}^{-} E_{F_{i+1}^{+}} & \\
=e^{-j(\alpha+\beta) \omega} c_{r_{l}+i}( & \left|A_{r_{l}+i}^{R}(\omega)\right|\left|B_{i}^{R}(\omega)\right| \\
& \left.-\left|A_{r_{l}+i+1}^{R}(\omega)\right|\left|B_{i+1}^{R}(\omega)\right|\right) .
\end{aligned}
$$

Obviously, if $\left|A_{r_{l}+i}^{R}(\omega)\right|\left|B_{i}^{R}(\omega)\right|=\left|A_{r_{l}+i+1}^{R}(\omega)\right|\left|B_{i+1}^{R}(\omega)\right|$, then the cross-term will be zero. This additional condition on $A_{r_{l}+i}(\omega)$ and $B_{i}(\omega)$ can be realized as the following matching condition:

$$
A_{r_{l}+i}(\omega)=B_{i}(\omega)
$$

or equivalently

$$
H_{r_{l}+i}\left(m_{l} \omega\right)=G_{i}(M \omega)
$$

$$
\begin{aligned}
D_{1}(\omega) & =\sum_{i=0}^{m_{l}-1} c_{r_{l}+i} A_{r_{l}+i}^{+}(\omega) B_{i}^{+}(\omega) \\
& =c_{r_{l}} A_{r_{l}}^{+}(\omega) B_{0}^{+}(\omega)+\cdots+c_{r_{l}+i} A_{r_{l}+i}^{+}(\omega) B_{i}^{+}(\omega)+\cdots+c_{r_{l}+m_{l}-1} A_{r_{l}+m_{l}-1}^{+}(\omega) B_{m_{l}-1}^{+}(\omega) \\
& =e^{-j(\alpha+\beta) \omega}\left(\begin{array}{r}
c_{r_{l}}\left|A_{r_{l}}^{R}(\omega)\right|\left|B_{0}^{R}(\omega)\right|-c_{r_{l}+1}\left|A_{r_{l}+1}^{R}(\omega)\right|\left|B_{1}^{R}(\omega)\right| \\
\quad+c_{r_{l}+2}\left|A_{r_{l}+2}^{R}(\omega)\right|\left|B_{2}^{R}(\omega)\right|-\cdots c_{r_{l}+m_{l}-1}\left|A_{r_{l}+m_{l}-1}^{R}(\omega)\right|\left|B_{m_{l}-1}^{R}(\omega)\right| \cdots
\end{array}\right) .
\end{aligned}
$$


This condition can be satisfied by properly choosing the filter lengths and setting the stopband and passband cutoff frequencies of the filters of the $M$-channel FB and $m_{l}$-channel TMUX. More details can be found in Section IV.

With the cross-term eliminated, $D_{2}(\omega)$ in (17) reduces to

$$
D_{2}(\omega)=c_{r_{l}} A_{r_{l}}^{+} B_{0}^{-} E_{F_{0}^{+}}+c_{r_{l}+m_{l}-1} A_{r_{l}+m_{l}-1}^{+} B_{m_{l}-1}^{-} E_{F_{m_{l}-1}^{-}} .
$$

This means that there is no bumping appear in the stopband of $\hat{H}_{l}(\omega)$. In addition, it can be easily verified that $\omega \in F_{0}^{+}$and $\omega \in F_{m-1}^{-}$ belong to the transition band of $\hat{H}_{l}(\omega)$.

From the above discussion, we can see that $c_{r_{l}+i}$ has acted simply as a sign indicator. For simplicity, we choose

$$
c_{r_{l}+i}=\left\{\begin{array}{l}
1, \quad \text { for even } i \\
-1, \quad \text { for odd } i
\end{array}\right.
$$

in the following discussion. Hence, the equivalent LTI filters can be written simply as follows:

$$
\hat{H}_{l}(z)=\sum_{i=0}^{m_{l}-1}(-1)^{i} H_{r_{l}+i}\left(z^{m_{l}}\right) G_{l, i}\left(z^{M}\right) .
$$

\section{DESIGN PROCEDURES AND EXAMPLES}

There are basically two main steps involved in the design of the proposed FB: a) designing the original FB and the recombination FB separately with the restriction of the existence and the matching conditions described in the last section and $b$ ) merging them accordingly. The detailed design procedures are summarized below.

1) Design the $M$-channel LP uniform FB and the $m_{l}$-channel LP recombination $\mathrm{FB}$ with alternate symmetry property. The design of the LP filter can be done by using the Parks-McClellan algorithm with the ideal magnitude response of the transition band having the form of cosine roll-off function.

2) Obtain the corresponding FB-based TMUX as $G_{l, i}(\omega)=$ $e^{-j \omega} G_{l, i_{\text {FB B }}}(\omega)$.

3) If all starting indexes $r_{l}$ are even, the subbands of the $M$-channel FB can be merged directly by using the synthesis filters of the $m_{l}$-channel TMUX.

4) If any $r_{l}$ is odd, the sequence $(-1)^{n}$ should be multiplied to the corresponding channels before merging the subbands.

5) Compensate for the delays introduced by the TMUXs in other branches of the $M$-channel uniform FB to make sure that the all branches experience the same amount of delay.

As mentioned previously, we want the magnitude response of the transition band of the analysis filters to follow a cosine roll-off characteristic. It makes sure that the sum of the square amplitude of two adjacent filters in their transition band is closed to unity so that the FB is approximately power complementary and is near-PR [9]. More specifically, for the $k$ th analysis filter $H_{k}(z)$ of the $M$-channel uniform $\mathrm{FB}$, the low-frequency transition band and the high-frequency transition band are expected to be, respectively

$$
\cos \left(\frac{\omega_{k_{-} p}^{l}-\omega}{\omega_{k_{-} p}^{l}-\omega_{k_{-} s}^{l}} \cdot \frac{\pi}{2}\right)
$$

and

$$
\cos \left(\frac{\omega-\omega_{k_{-} p}^{h}}{\omega_{k_{-}}^{h}-\omega_{k_{-} p}^{h}} \cdot \frac{\pi}{2}\right) .
$$

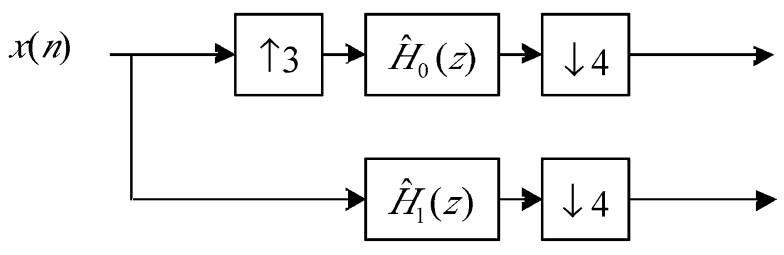

(a)

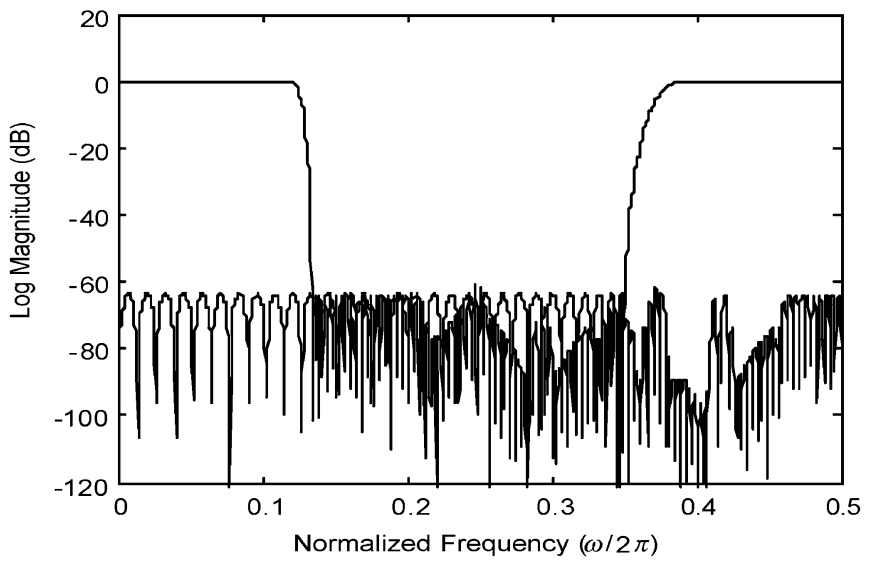

(b)

Fig. 2. Linear-phase recombination nonuniform filter bank with sampling factors $(3 / 4,1 / 4)$. (a) Equivalent structure of the analysis banks with LTI analysis filter. (b) Magnitude responses of $\hat{H}_{0}(\omega)$ (low-pass filter) and $\hat{H}_{1}(\omega)$ (high-pass filter).

Notice that only the specifications for positive frequencies are given since the magnitude function is even. Here, $\omega_{k_{-} p}^{l}$ and $\omega_{k_{-} s}^{l}$ are the passband and stopband cutoff frequencies of the low-frequency transition band; and $\omega_{k_{-} p}^{h}$ and $\omega_{k_{-} s}^{h}$ are the passband and stopband cutoff frequencies of the high-frequency transition band. We have $\omega_{k_{-} s}^{l}<\omega_{k_{-} p}^{l}<$ $\omega_{k_{-} p}^{h}<\omega_{k_{-} s}^{h}$ and the transition bandwidth being $\Delta \omega=\omega_{k_{-} p}^{l}-$ $\omega_{k_{-} s}^{l}=\omega_{k_{-} s}^{h}-\omega_{k_{-} p}^{h}$. For the low-pass filter $H_{0}(z)$ and high-pass filter $H_{M-1}(z)$, there is only one transition band for each: a high-frequency transition band for $H_{0}(z)$ and a low-frequency transition band for $H_{M-1}(z)$. The cutoff frequencies of all the filters of $M$-channel FB are as follows:

$$
\begin{aligned}
& \omega_{k_{-} p}^{l}=(k \pi / M)+(\Delta \omega / 2)=\omega_{k-1 \_s}^{h}, \\
& \omega_{k_{-} s}^{l}=(k \pi / M)-(\Delta \omega / 2)=\omega_{k-1 \_p}^{h}, \quad k=1, \ldots, M-1 .
\end{aligned}
$$

The design for the LP uniform FBs and TMUXs can be done by using the Parks-McClellan algorithm in Matlab, where the relative weighting of the passband and stopband errors are chosen to be identical.

We will now present two design examples based on the method described above. To show flexibility in choosing numbers of channels of the FB and TMUX, we give the two examples being with different $M$ and $m_{l}$ (even and odd). In these examples, we assume that the uniform LP FBs have equal filter lengths for simplicity.

Example 1: Consider an LP RNFB with sampling factors $(3 / 4,1 / 4)$. It is constructed by merging the first three channels of a four-channel uniform LP FB with a three-channel recombination LP TMUX. In the original FBs $H_{0}(z)$ and $H_{2}(z)$ are chosen to be symmetric and $H_{1}(z)$ and $H_{3}(z)$ are antisymmetric. In the recombination TMUX, $G_{0}(z)$ and $G_{2}(z)$ are chosen to be symmetric, while $G_{1}(z)$ is antisymmetric. As mentioned earlier, the use of a cosine-roll-off transition band allows us to solve the filter design 


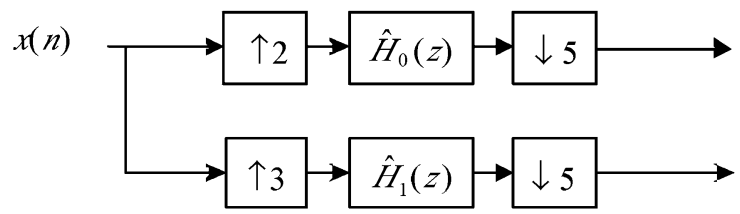

(a)

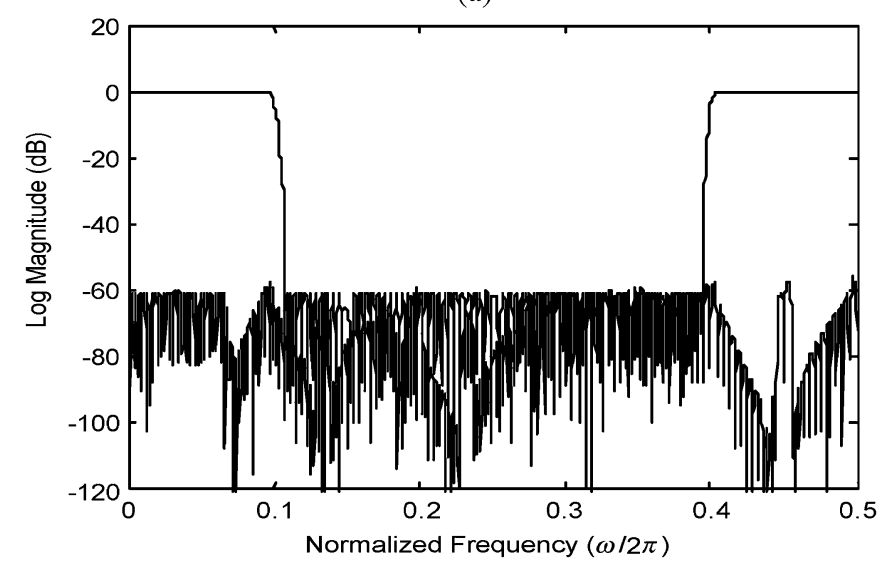

(b)

Fig. 3. Linear-phase recombination nonuniform filter bank with sampling factors $(2 / 5,3 / 5)$. (a) Equivalent structure of the analysis banks with LTI analysis filter. (b) Magnitude responses of $\hat{H}_{0}(\omega)$ (low-pass filter) and $\hat{H}_{1}(\omega)$ (high-pass filter).

problem by using the Parks-McClellan algorithm in Matlab. In addition, the matching condition in (23) is much easier to be satisfied by choosing $N_{M} / M=N_{m_{l}} / m_{l}$. As an example, we choose the lengths of the three- and four-channel FBs to be 63 and 84, respectively. In this example, according to (27), the passband and stopband cutoff frequencies of the analysis filters of the four-channel original $\mathrm{FB}$ are as follows: for $H_{0}(z), \omega_{0 \_p}^{h}=0.1 \times(2 \pi), \omega_{0_{-} s}^{h}=0.15 \times(2 \pi)$; for $H_{1}(z), \omega_{1 \_s}^{l}=0.1 \times(2 \pi), \omega_{1-p}^{l}=0.15 \times(2 \pi), \omega_{1-p}^{h}=0.225 \times(2 \pi)$ and $\omega_{1_{-} s}^{h}=0.275 \times(2 \pi)$. The cutoff frequencies of the other analysis filters can be defined similarly. The passband and stopband cutoff frequencies of the synthesis filters of the three-channel recombination TMUX are set similar to that of the $M$-channel FB with $m_{l}$ replacing $M$ in (27). Here, we have $m_{0}=3$. Following the design procedures described above, we obtained the RNFB. The equivalent structure of the FB with the resulting LTI analysis filters is shown in Fig. 2(a). The magnitude responses of the equivalent LTI analysis filters $\hat{H}_{0}(\omega)$ (low-pass filter) and $\hat{H}_{1}(\omega)$ (high-pass filter) are shown in Fig. 2(b). In this example, $\hat{H}_{1}(\omega)=H_{3}(\omega)$, where $H_{3}(\omega)$ is the original high-pass filter of the four-channel uniform FBs since no merging operation in this channel of the original uniform FB. This system is approximately $\mathrm{PR}$ and the reconstruction error is about $10^{-3}$ and the aliasing errors is also about $10^{-3}$.

Example 2: This example is an LP RNFB with sampling factors $(2 / 5,3 / 5)$. By the above design procedure, we obtain the equivalent LTI analysis filters as shown in Fig. 3. The lengths of the two-, three-, and five-channel FBs are 50,75, and 125, respectively. The reconstruction error is about $10^{-3}$, and the aliasing errors is also about $10^{-3}$.

It can be seen from the above examples that the design of the proposed near-PR LP RNFBs is very simple, and good filter quality is achieved.

\section{CONCLUSION}

This correspondence presented a novel design method of LP RNFBs. By analyzing the properties of the analysis filters of the RNFB, we derived the existence and the matching conditions so as to obtain LP RNFBs. Based on the obtained conditions, we proposed a design method that is very simple but with good frequency characteristics as demonstrated by two design examples.

\section{REFERENCES}

[1] P. Q. Hoang and P. P. Vaidyanathan, "Non-uniform multirate filter banks: Theory and design," Proc. IEEE Int. Symp. Circuits Systems, vol. 1, pp. 371-374, May 1989.

[2] J. Kovacevic and M. Vetterli, "Perfect reconstruction filter banks with rational sampling factors," IEEE Trans. Signal Process., vol. 41, no. 6, pp. 2047-2066, Jun. 1993.

[3] R. V. Cox, "The design of uniformly and nonuniformly spaced pseudo quadrature mirror filters," IEEE Trans. Acoust., Speech, Signal Processing, vol. 34, no. 5, pp. 1090-1096, Oct. 1986.

[4] S. C. Chan, X. M. Xie, and T. I. Yuk, "Theory and design of a class of cosine-modulated nonuniform filter banks," in Proc. IEEE Int. Conf. Acoustics, Speech, Signal Processing (ICASSP), vol. 1, Jun. 2000, pp. 504-507.

[5] X. M. Xie, "New Design and Realization methods for perfect reconstruction nonuniform filter banks," Ph.D. dissertation, Elect. and Electronic Eng. Dept., The University of Hong Kong, Hong Kong, China, Jan. 2004.

[6] O. A. Niamut and R. Heusdens, "Subband merging in cosine-modulated filter banks," IEEE Signal Process. Lett., vol. 10, no. 4, pp. 111-114, Apr. 2003.

[7] A. K. Soman, P. P. Vaidyanathan, and T. Q. Nguyen, "Linear-phase paraunitary filter banks: Theory, factorizations and applications," IEEE Trans. Signal Process., vol. 41, no. 12, pp. 3480-3496, Dec. 1993.

[8] Y. P. Lin and P. P. Vaidyanathan, "Linear phase cosine modulated maximally decimated filter banks with perfect reconstruction," IEEE Trans. Signal Process., vol. 42, no. 11, pp. 2525-2539, Nov. 1995.

[9] P. P. Vaidyanathan, Multirate Systems and Filter Banks. Englewood Cliffs, NJ: Prentice-Hall, 1993.

[10] R. D. Koilpillai, T. Q. Nguyen, and P. P. Vaidyanathan, "Some results in the theory of crosstalk-free transmultiplexers," IEEE Trans. Signal Process., vol. 39, no. 10, pp. 2174-2183, Oct. 1991. 